K. Wallenfang

R. Stadler

\section{Wundheilungskonzept mit heute zur Verfügung stehenden Wundverbänden zur phasenadaptierten Wundbehandlung}

Wound Management with Currently Available Wound Dressing for Phase

Adapted Treatment
Zusammenfassung

Die phasenadaptierte moderne Wundbehandlung bietet die Möglichkeit einer raschen, komplikationslosen Wundheilung akuter und chronischer Wunden. Bei der Auswahl eines geeigneten Wunddressings müssen das Stadium der Wundheilung sowie das Ausmaß von Nekrosen, Belägen, Exsudation und Superinfektion berücksichtigt werden. Bei der Vielzahl heute zur Verfügung stehender Wundbehandlungen soll diese Arbeit ein praxisrelevantes Konzept bieten, um phasengerechte, effektive Wundtherapie durchführen zu können.

\section{Einleitung}

Ziel einer jeden Wundbehandlung ist die Unterstützung oder Wiederherstellung der physiologischen Wundheilung durch Beseitigung von Störfaktoren und Mangelzuständen.

Die Genese chronischer Wunden ist vielfältig. Am häufigsten sind vaskuläre Ursachen wie venöse Insuffizienz (z. B. Ulcus cruris venosum), arterielle Verschlusskrankheit (z. B. Ulcus cruris arteriosum, Dekubitus) und diabetische Angiopathie (z. B. diabetische Gangrän, Dekubitus), die auch in Kombination auftreten können. Seltenere Ursachen sind Infektionen (z. B. Erysipel), hämatopoetische Erkrankungen (z.B. Sichelzellanämie), immunologische Erkrankungen (z.B. Vaskulitis, Pyoderma gangraenosum), Neoplasien (z.B. Plattenepithelkarzinom), neurologische Erkrankungen (z. B. Poliomyelitis) und Trauma.

Die Therapie chronischer Wunden stellt sich oft als schwierig und frustran heraus, nicht zuletzt aufgrund der häufigen Ratlosigkeit gegenüber dem großen Angebot heute zur Verfügung stehender Wundtherapeutika. Die richtige Auswahl der im jeweiligen Fall geeignetsten Wundbehandlung erfordert ein hohes Maß
Abstract

Modern and phase adapted wound management offers the possibility of achieving fast wound healing of acute and chronic wounds without complications. The stage of wound healing as well as the extent of necrosis, coating, exsudation, and superinfections have to be considered on choosing an adequate wound dressing. As the number of available wound treatments nowadays is very extensive this paper is to be used as a concept for out-patient surgeries to apply phase adapted and effective wound therapy.

an Erfahrung und die genaue Kenntnis der zur Verfügung stehenden Möglichkeiten. Um eine optimale Versorgung chronischer Wunden zu gewährleisten, sind in zunehmendem Maße Einrichtungen mit interdisziplinär organisierten Wundheilungssprechstunden erforderlich. In sog. Wundheilungssprechstunden sollte die lokale Wundbehandlung sowie die Koordination der einzelnen interdisziplinären Behandlungsschritte vorgenommen werden. Ausführende Kraft könnten ein Facharzt, ein Assistenzarzt und ein bis mehrere Pflegekräfte sein. Durch Zusammenarbeit mit Hausärzten, Sozialstationen und ambulanten Pflegediensten wird die Infrastruktur erweitert, wodurch eine ambulante Behandlung unter kontrollierten Bedingungen möglich ist. Hierdurch können lange stationäre Aufenthalte mit hohen Kosten reduziert und resultierende Risiken wie Arbeitsunfähigkeit, Arbeitsplatzverlust oder Frühberentung vermindert werden. Die Indikation zur stationären Behandlung sollte als Alternative zur ambulanten Behandlung im Rahmen einer Wundheilungssprechstunde streng definiert werden; z.B. zu erweiterten chirurgischen Eingriffen oder bei schwerer Wundinfektion. 
Um eine phasengerechte Wundbehandlung zu gewährleisten, sind vor und während Therapie regelmäßig Wundinspektionen vorzunehmen. Neben Wundlokalisation und Wundgröße sind klinischer Aspekt, Stadium der Wundheilung, Ausmaß von Exsudation und das Vorhandensein von Belägen, Nekrosen oder Superinfektionen wichtige Parameter zur Aufstellung eines geeigneten phasenadaptierten Wundheilungskonzeptes. Zur Qualitätskontrolle sollte ein EDV-gestütztes Dokumentationssystem erarbeitet werden.

An einen optimalen Wundverband sind zahlreiche Anforderungen zu stellen: Aufnahme von überflüssigem Wundsekret ohne Wundaustrocknung mit der Möglichkeit atraumatischer Verbandswechsel; steriles, nichttoxisches und nichtsensibilisierendes Material; Adaptationsfähigkeit an die in der Wunde herrschenden Heilungsphasen; Gasaustausch Verband/Umwelt muss begrenzt möglich sein; Schutz gegenüber physikalischen, chemischen und mikrobiellen Belastungen; Reduktion von Schmerz und Juckreiz; keine Inkorporation von Verbandsbestandteilen in die Wunde; Eignung zur Selbstbehandlung durch den Patienten; gutes Preis-Wirksamkeits-Verhältnis [21].

Grundlage der modernen Wundbehandlung ist das Prinzip der feuchten Wundheilung. Feuchte Wundheilung heißt, dass in produktabhängigem Ausmaß Wundsekret durch Semiokklusion oder Absorption in bzw. unter der Wundauflage retiniert wird. Dieses Wundsekret enthält im Feuchtmilieu mehr zelluläre Bestandteile und diverse Wachstumsfaktoren. Zellaktivitäten wie Chemotaxis, mitotische Aktivität, Angiogenese, Matrixformation, dermale Reparation, Keratinozytenmigration und autolytisches Débridement werden stimuliert [4].

Im Folgenden soll ein Überblick über die Möglichkeiten phasenadaptierter, moderner Wundbehandlungen gegeben werden:

\section{Wundreinigung}

Im Vordergrund steht das chirurgische Wunddébridement. Dabei werden Fremdkörper, nekrotisches oder infiziertes Gewebe und schmierige Beläge entfernt, Wundtaschen werden, wenn nötig, eröffnet und es wird für saubere und glatt begrenzte Wundränder gesorgt. Eine enzymatische Wundreinigung kann das chirurgische Débridement unterstützen und ergänzen.

Eine Alternative zur chirurgischen Wundreinigung stellt die Biochirurgie dar. „Biochirurgie“ bedeutet das Aufbringen von sterilen Fliegenmaden auf nekrotisches Gewebe mit dem Ziel der Wundreinigung. Im Wesentlichen werden heutzutage die Maden der Goldfliege Lucilia sericata eingesetzt. Es handelt sich um reine Nekrophagen („Biosurgeons“), so dass eine Schädigung gesunden Gewebes ausgeschlossen ist. Im Rahmen der extrakorporalen Verdauung wird abgestorbenes Gewebe durch proteolytische Enzyme verflüssigt und gleichzeitig ein hochgradig mikrobizides Wundmilieu geschaffen. Eine starke antimikrobielle Aktivität konnte gegen Staphylococcus aureus nachgewiesen werden. Etwas weniger, aber immer noch deutlich empfindlich reagieren Pseudomonas sp. und MRSA. Keine Hemmung des Bakterienrasens ist für E. coli, Enterococcus und Proteus nachgewiesen. Durch die mechanische Irritation der umherkriechenden Maden wird einerseits die Wundexsudation angeregt, wodurch nekroti- sches Gewebe und Bakterien ausgespült werden, andererseits die Bildung von Granulationsgewebe gefördert. Die Applikation der Lucilia-Larven auf Wunden kann für den Patienten schmerzhaft sein, vor allem wenn eine bereits spontan schmerzhafte Wunde vorliegt. Die Schmerzen werden der Wirkung der potenten proteolytischen Enzyme, der mittels der Mundhaken bewerkstelligten lebhaften Lokomotion der Larven auf der Wunde und der Reizung der Wunde durch die zahnartigen Auswüchse der Larvenhaut zugeschrieben. Nichtsdestotrotz ist im Gegensatz zur operativen Nekrosenabtragung keine Anästhesie erforderlich, treten keine Blutungen auf und die lokale Stoffwechselsituation wird durch iatrogene Wundsetzung nicht weiter verschlechtert. Die Applikation ist relativ einfach. Die sterilen Larven werden auf die Wunde aufgebracht, durch ein feinmaschiges steriles Netz abgedeckt, damit sie nicht entweichen können und nach 3 Tagen wieder ausgespült. Neuerdings stehen auch sog. „Biobags“ zur Verfügung. Hierbei werden die Larven in dünne Polyvinylalkohol-Folien verpackt, welche direkt auf die oberflächliche oder tiefe Wunde gelegt werden. Der Austausch von Enzymen und verflüssigtem nekrotischen Gewebe als Nährsubstrat der Larven bleibt gewährleistet. Die Applikation ist wesentlich vereinfacht, ein Entweichen der Larven ist ausgeschlossen, die Aktivität der Larven kann leichter kontrolliert werden und das biochirurgische Débridement kann gezielter eingesetzt werden. Die Behandlung kann bis zur vollständigen Wundreinigung mehrmals wiederholt werden und anschließend auf eine granulations- bzw. epithelisierungsfördernde moderne Wundauflage umgestellt werden. Indikationen sind Ulcera crurum und Dekubitalulzerationen, insbesondere bei multimorbiden und nicht narkosefähigen Patienten, die therapieresistente diabetische und ischämische Fußgangrän und areaktive Wunden $[2,15,20,25]$.

Moderne Wundauflagen in der Exsudations-/Reinigungsphase, Granulationsphase und Epithelisierungsphase (Tab. 1 u. 2 )

Ausdruck der körpereigenen, aktiven Wundreinigung ist die Entstehung des Wundödems. Die daraus resultierende starke Exsudatbildung unterstützt die Wundsäuberung durch die in das Wundgebiet einwandernden Leukozyten, Monozyten und Makrophagen. Aufgelöster Wundschorf, Zelltrümmer, Fremdkörper und Bakterien werden ausgeschwemmt. In dieser Phase muss der geeignete Wundverband sehr saugfähig sein, trotzdem soll die Wundoberfläche feucht gehalten werden. Mazerationen sollen vermieden werden.

Nach Abschluss der Reinigungsphase beginnt die eigentliche Reparaturphase. Aus dem gesunden Nachbargewebe sprossen neue Gefäße in das Wundgebiet ein. Fibroblasten und Endothelzellen proliferieren. Der Wundgrund füllt sich mit dunkelrotem Granulationsgewebe. In dieser Phase darf die Wunde nicht austrocknen. Die optimale Wundabdeckung muss vorhandenes Wundsekret aufsaugen und gleichzeitig die Wundoberfläche feucht halten. Es sollte ein Wasserdampf- und Sauerstoffaustausch gewährleistet sein und die Wunde vor eindringenden Keimen geschützt werden.

In der Epithelisierungsphase bilden die Fibroblasten vermehrt Kollagen und Proteoglykane für den Aufbau des Narbengewebes. Die Wundkontraktion setzt ein. Vom Wundrand, bei flachen 
Tab. 1 Indikationen der einzelnen Wundauflagen

\begin{tabular}{|c|c|}
\hline Stadium der Wundheilung & Art der Wundauflage \\
\hline Nekrose (Abb. 1) & $\begin{array}{l}\text { Aktivkohleverbände } \\
\text { Hydrogele } \\
\text { Gele }\end{array}$ \\
\hline (Super-)Infektion & $\begin{array}{l}\text { Alginate } \\
\text { Aktivkohleverbände } \\
\text { Polyurethan-Schaumstoffe }\end{array}$ \\
\hline Beläge (Abb. 2) & $\begin{array}{l}\text { Alginate } \\
\text { Aktivkohleverbände } \\
\text { Hydrofaser } \\
\text { Hydrogele } \\
\text { Gele } \\
\text { Hydrokolloide } \\
\text { Polyurethan-Schaumstoffe }\end{array}$ \\
\hline Exsudation & $\begin{array}{l}\text { Alginate } \\
\text { Aktivkohleverbände } \\
\text { Hydrofaser } \\
\text { Polyurethan-Schaumstoffe }\end{array}$ \\
\hline Granulation (Abb. 3) & $\begin{array}{l}\text { Hydrofaser } \\
\text { Hydrogele } \\
\text { Gele } \\
\text { Hydrokolloide } \\
\text { Polyurethan-Schaumstoffe, Hydropolymere }\end{array}$ \\
\hline Epithelisierung (Abb. 4) & $\begin{array}{l}\text { Hydrokolloide } \\
\text { Hydrogele } \\
\text { Hydropolymere } \\
\text { nichthaftende feuchte Wundauflagen } \\
\text { Polyurethanfilme }\end{array}$ \\
\hline
\end{tabular}

Wunden auch vom Wundgrund aus, wandern die Epithelzellen konzentrisch zur Mitte hin. In diesem Stadium hat die Wundabdeckung die Aufgabe, das empfindliche neugebildete Gewebe zu schützen und vor äußeren Einflüssen abzuschirmen $[4,6]$.

\section{Alginate}

Alginate bestehen aus zu Fasern versponnenen Natrium-Kalzium-Salzen der aus marinen Braunalgen gewonnenen Alginsäure. Bei Kontakt mit Wundsekret werden durch Austausch von Natrium- und Kalziumionen die unlöslichen Fasern in lösliches Natrium-Kalzium-Alginat umgewandelt. Aus diesem Mechanismus resultiert eine Art Gelbildung des zunächst faserigen Verbandsmaterials. Durch Gelbildung wird ein feuchtes Wundmilieu geschaffen, die Wunde wird vor dem Austrocknen geschützt. Alginate sind sowohl als Kompresse als auch als Tamponade im Handel und damit sowohl für flache Wunden als auch tiefe Wundhöhlen geeignet $[16,17]$.

Aufgrund der großen Sekretaufnahmekapazität und der Eigenschaft, gleichzeitig Zelldetritus und Bakterien zu absorbieren, sind Alginate ideal zur Behandlung stark sezernierender, nässender sowie verschmutzter und infizierter Wunden, einschließlich Wunden mit stark zerklüftetem Grund (z. B. Dekubitalulzera) [3]. Alginate eignen sich auch zur Behandlung blutender Wunden. Die relative Kontraindikation bei trockenen Wunden kann durch Anfeuchten der Kompresse oder Tamponade mit steriler Kochsalzlösung umgangen werden und damit das Indikationsspektrum dieses wirkungsvollen Wunddressings erweitert werden.
Tab. 2 Hauptgruppen moderner Wundauflagen mit Produktbeispielen

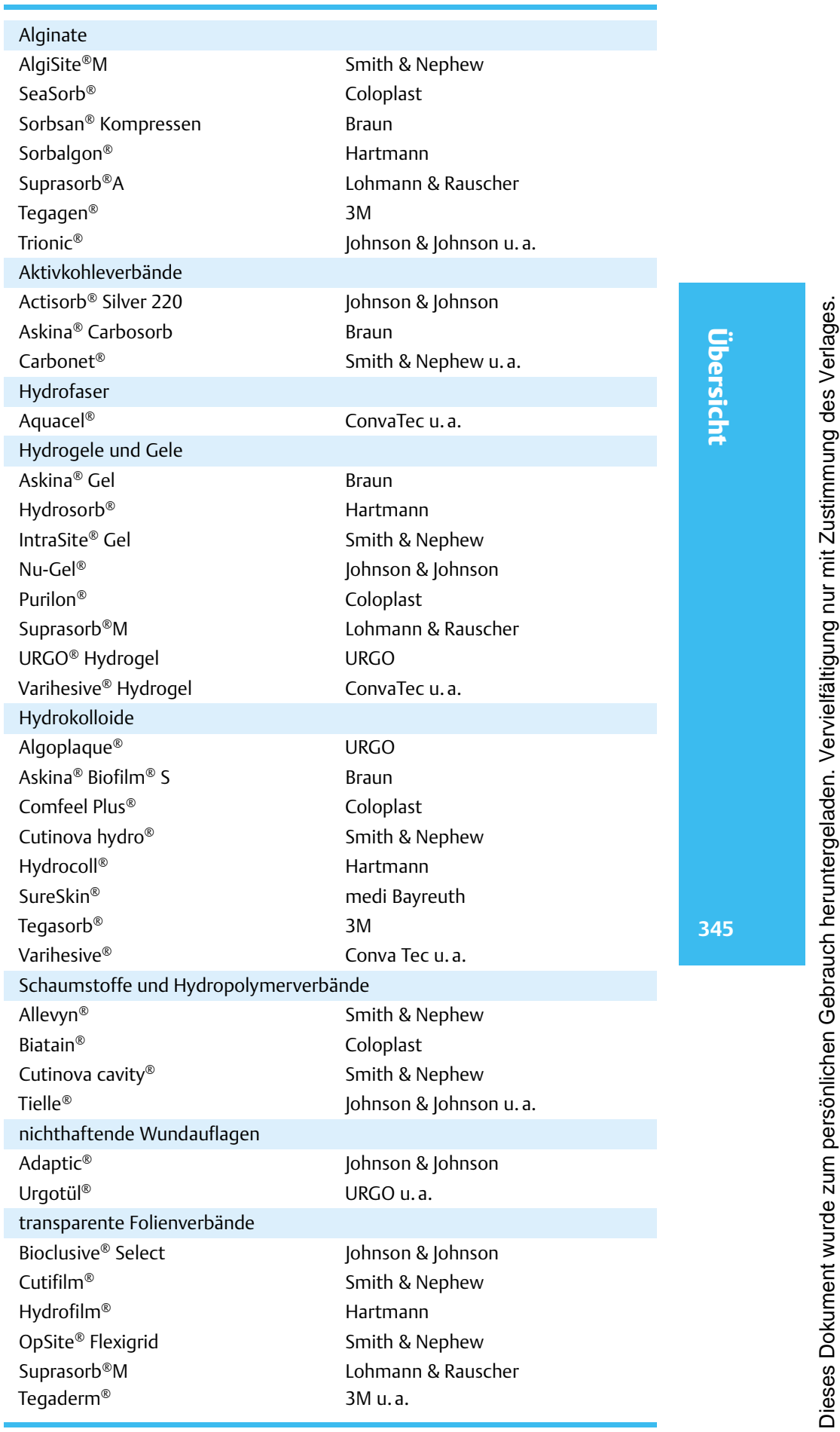

\section{Aktivkohleverbände}

Aktivkohlehaltige Wundauflagen folgen ausnahmsweise nicht dem Prinzip der feuchten Wundheilung. Ihr wundheilungsfördernder Einfluss beruht auf ihren physikalischen Eigenschaften. Sie bestehen aus einem Aktivkohlegewirk, das von einem Polyamid-Vlies umschlossen ist, und sie können silberimprägniert sein. Aus der sehr großen Oberfläche des Fasergewirks resultiert eine hohe Absorptionskapazität für Bakterien, Sekret, Zelldetritus sowie der für üblen Wundgeruch verantwortlichen Diamine, 


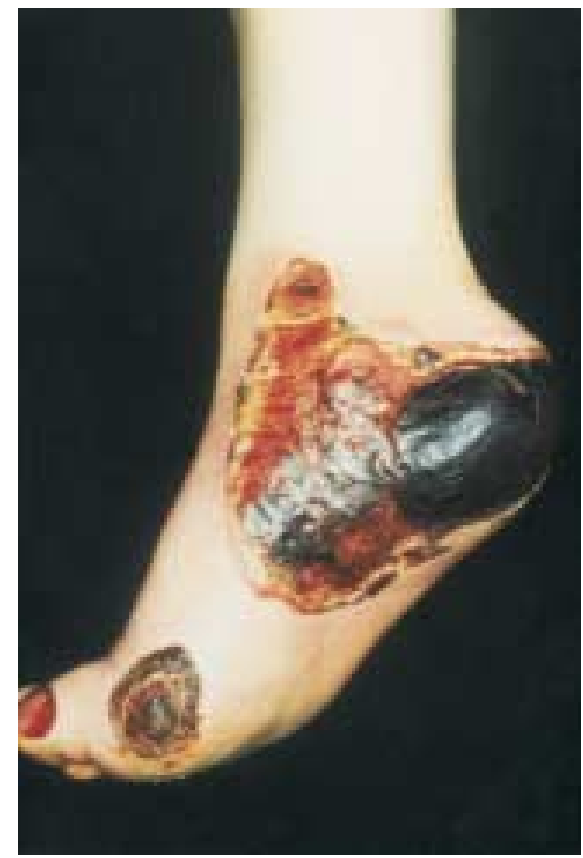

Abb. 1 Ulkus mit großflächiger Nekrose.

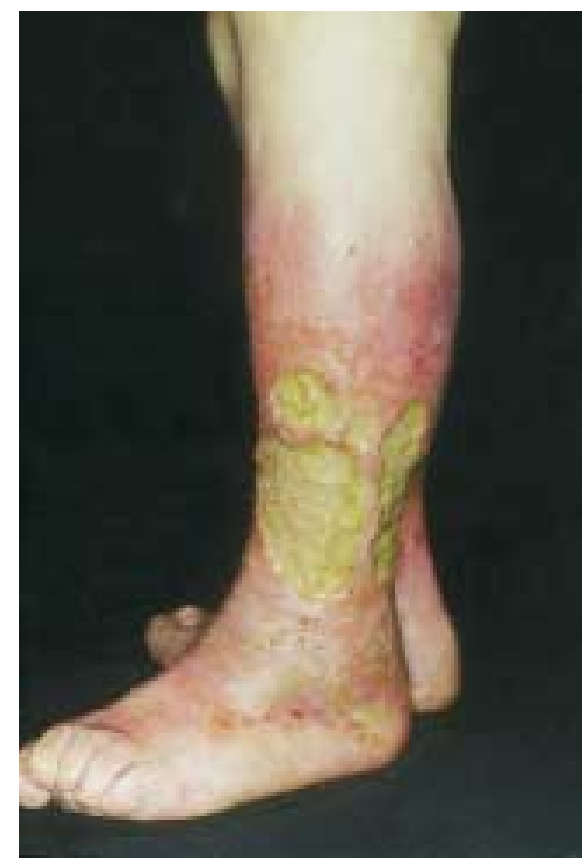

Abb. 2 Ulkus mit schmierig-gelblichem Belag.

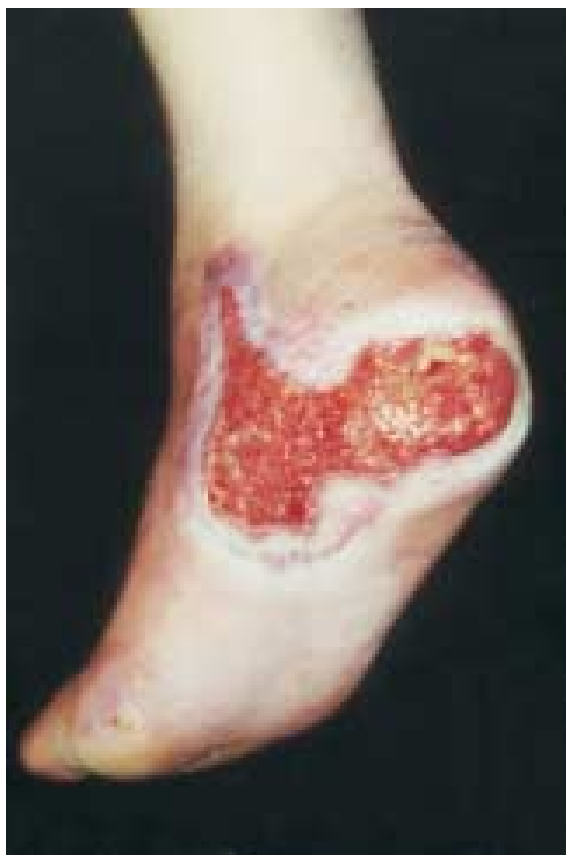

Abb. 3 Ulkus mit roter sauberer Granulation und beginnender Epithelisierung vom Randbereich.

Putrescine und Kadaverine. Durch Silberimprägnierung kann die antimikrobielle Wirkung noch gesteigert werden [19].

Wichtig ist, dass aktivkohlehaltige Wunddressings nur temporär bei exsudativen, infizierten und übel riechenden Wunden, dagegen nicht bei trockenen Wunden eingesetzt werden dürfen, da dann durch das fehlende Wundsekret kein Kontakt zwischen Wunde und Wundauflage zustande kommen kann. Sollte der oft schmerzhafte Verbandswechsel vom Patienten nicht toleriert werden, kann direkt auf die Wunde eine nichthaftende vaselinimprägnierte Wundgaze appliziert werden oder der aktivkohlehaltige Tebbe-Verband mit Ringerlösung angefeuchtet werden. Zur Fixierung ist ein Sekundärverband erforderlich.

\section{Hydrofaser}

Das aus makromolekularer Natriumcarboxymethylzellulose bestehende Material verwandelt sich bei der Absorption von Wundexsudat in ein formstabiles Gel. Die Absorptionsfähigkeit der Hydrofaser ist sehr hoch, wobei eine horizontale Ausbreitung der Flüssigkeit im Material minimal ausfällt und somit Wundrandmazerationen vermieden werden [1].

Indikationen sind stark bis sehr stark exsudierende, auch tiefe Wunden in der Exsudations- und Granulationsphase. Hydrofaserverbände eignen sich auch für infizierte Wunden [3].

\section{Hydrogele}

Hydrogele sind dreidimensionale Netzwerke aus hydrophilen Polymeren, die einen unterschiedlichen Prozentsatz an Wasser beinhalten, selbst aber unlöslich sind. Durch Ausdehnung der Querverbindungen der Polymerketten können Flüssigkeit und darin enthaltene Makromoleküle aufgenommen werden. Die wundferne semipermeable Polyurethanfolie sorgt für die Aufrechterhaltung eines feuchten Wundmilieus und dient als Barriere zur Außenwelt [14]. Die semitransparenten Eigenschaften ermöglichen auch bei mehrtägiger Tragedauer eine Wundin-

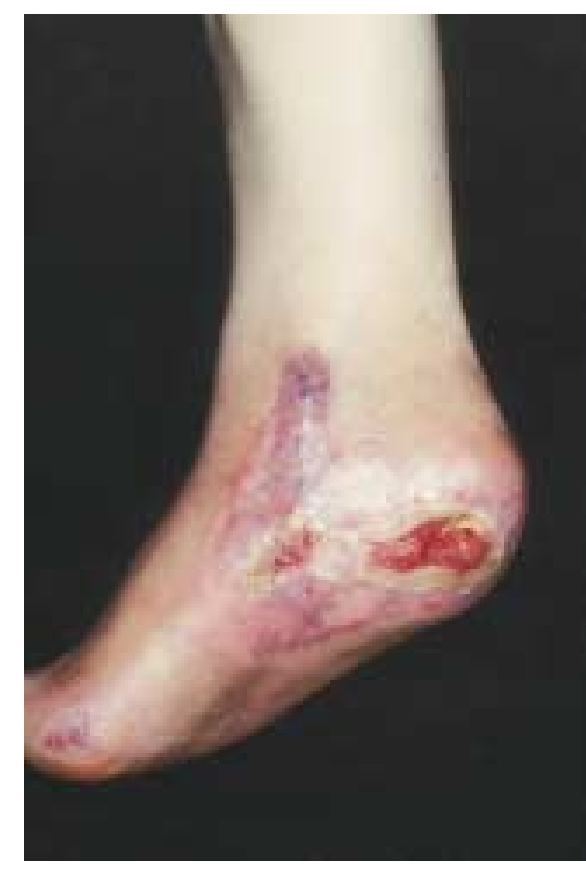

Abb. 4 Nahezu abgeheiltes Ulkus mit guter Epithelisierungstendenz.

spektion durch den Verband hindurch. Vom Patienten werden die Hydrogele als angenehm kühlend empfunden.

Haupteigenschaft der Hydrogele ist aufgrund des hohen Wasseranteils das Aufweichen von Nekrosen und Belägen. Indikationen sind fibrinbelegte Wunden, trockene verschorfte Wunden, Schürfwunden und Spalthautentnahmestellen. Neben der Reinigungsphase können Hydrogele auch phasenübergreifend in der Granulations- und Epithelisierungsphase eingesetzt werden.

Gele

Gele bestehen aus einem viskösen, klaren Gel aus Wasser, Pektin und Natriumcarboxymethylzellulose mit hydrokolloidalen Bestandteilen. Ihnen kann Alginat zugesetzt sein. Mögliche anti- 
mikrobielle Eigenschaften beruhen auf den teilweise enthaltenen längerkettigen Alkoholen. Gele führen durch Rehydratisierung trockenen Gewebes ähnlich wie Hydrogele zum Lösen von Nekrosen und Fibrinbelägen, wodurch ein schonendes Débridement möglich wird. Die hydrokolloidalen Bestandteile bedingen eine gewisse Sekretaufnahmekapazität, zugesetztes Alginat wirkt zusätzlich wundheilungsfördernd [21].

Hauptindikation sind nekrotische und belegte, insbesondere trockene Wunden.

\section{Hydrokolloide}

Hydrokolloide Wundauflagen sind durch einen zweischichtigen Aufbau gekennzeichnet. Wundkontakt hat eine selbsthaftende Schicht aus hydrokolloidalen, flüssigkeitsabsorbierenden Mikrogranula (meist mit Carboxymethylzellulose, Polysacchariden und Proteinen), wundfern liegt eine wasserabweisende, semiokklusive, vor sekundärer Kontamination schützende Trägerschicht aus Polyurethanfolie.

Bei Aufnahme von Wundsekret in die hydrophilen Mikrogranula entsteht ein Gel, wodurch sich die adhäsiven Eigenschaften des Hydrokolloids über der Wundfläche verlieren. Dies wird auch als Phasenumkehr bezeichnet. Durch die Phasenumkehr wird ein atraumatischer Verbandswechsel gewährleistet. Das unter Hydrokolloiden gebildete Gel riecht oft übel und darf nicht mit Pus verwechselt werden. Die Tragedauer richtet sich nach der Exsudatmenge der Wunde. Ein Auslaufen von Sekret am Rand des Verbandes signalisiert die Notwendigkeit des Verbandswechsels. Gelrückstände müssen bei jedem Verbandwechsel entfernt werden.

Das Gelieren und Anschwellen der hydrokolloidalen Schicht trägt zur Erhaltung eines feuchten Wundmilieus und damit zu einer schnelleren Wundheilung bei. Eine zu Therapiebeginn scheinbare Vergrößerung der Wunde ist eine natürliche Folge der Gewebshydratation unter Semiokklusion und der damit einhergehenden autolytischen Wundreinigung und Demarkation.

\section{Selten werden allergische Kontaktdermatitiden durch Kolopho-} niumderivate der Klebeschicht beschrieben.

Indikationen für Hydrokolloide sind granulierende Wunden mit nur geringer bakterieller Besiedlung und bei nicht zu starker Exsudation. Bei zu geringer Wundexsudation kann keine Phasenumkehr stattfinden und eine Traumatisierung des Wundbettes durch Abreißen oberflächlicher Hautschichten resultiert.

Dünnere Platten können als Schutz der Wunde in der Granulationsphase oder als vorübergehender steriler Wundverschluss von nicht primär schließbaren Wunden dienen. Durch ihre hydroaktiven Eigenschaften sind Hydrokolloide auch in der Lage, fibrinöse schmierige Beläge aufzuweichen und zu lösen [10,14].

Kontraindikationen sind stark infizierte Wunden, freiliegende Sehnen und Knochen, Wunden mit starker Exsudation und Ulzerationen mit fehlender Granulationstendenz.

\section{Schaumstoffe}

Schaumstoffe sind zumeist dreischichtig aufgebaut. Wundkontakt hat ein nichthaftendes Strukturgitter, das das Einwachsen von Granulationsgewebe bei mehrtägigem Verbleib auf der Wunde verhindern soll. In der Mitte liegt der offenporige absorbierende Polyurethanschaumstoff. Die wundferne Schicht wird von einer semiokklusiven Polyurethanfolie gebildet.

Hauptfunktion der Schaumstoffe ist in produktabhängigem Ausmaß die Absorption von Exsudat, Pus und Zelldetritus, die auf Kapillarkräften des Polyurethanschaumstoffes beruht. In ihrer Eigenschaft der Granulationsförderung werden die Schaumstoffe häufig auch als Bereiter von gut vaskularisierten und granulierten Hauttransplantatbetten eingesetzt [13].

Hydropolymere, eine Sondergruppe mit vielen Ähnlichkeiten zu Schaumstoffen, werden auch gerne in der Epithelisierungsphase eingesetzt.

Kontraindikation für Polyurethanschaumstoffauflagen sind trockene und stark superinfizierte Wunden.

\section{Nichthaftende Wundauflagen}

Nichthaftende Wundauflagen bestehen aus einem Fasergewirk aus Baumwolle oder Viskose und können wirkstofffrei (Öl-inWasser-Emulsion) oder wirkstoffhaltig (PVP-Jod, Chlorhexidin, Fusidinsäure) imprägniert sein. Hierbei besteht das Risiko einer möglichen Kontaktallergie, einer Verzögerung der Wundheilung und einer Resistenzbildung der Erreger [11,23].

Nichthaftende Wundauflagen verhindern ein Verkleben des Sekundärverbandes mit der Wunde und ermöglichen hierdurch atraumatische Verbandswechsel. Einsatzbereiche sind frische Wundnähte und epithelisierende Wunden wie Spalthautentnahmestellen, Schürf- und Verbrennungswunden.

\section{Transparente Folienverbände}

Transparente Folienverbände bestehen aus einem dünnen Polyurethanfilm mit selbsthaftender Acrylat- oder Vinylätherbeschichtung. Sie sind semipermeabel, d.h. luft- und wasserdampfdurchlässig, jedoch impermeabel für Wasser und Mikroorganismen [24]. Die Transparenz ermöglicht jederzeit eine Wundinspektion. Sie werden als Inzisionsfolie bei Operationen, als transparenter Verband von Venenkathetern, zur okklusiven Salbentherapie und in der Epithelisierungsphase gering nässender Wunden eingesetzt. Nicht zuletzt dienen sie auch als wasserabweisende Wundabdeckung beim Duschen.

\section{Vakuumversiegelung}

Die Vakuumversiegelung ist eine Wundbehandlungsmethode, bei der ein subatmosphärischer Druck auf die Wundoberfläche einwirkt. Dazu wird ein Polyvinylalkoholschwamm mit eingelegtem Drainagesystem auf die Wunde aufgelegt, mit einer transparenten Verbandsfolie hermetisch abgeklebt und an eine Vakuumquelle angeschlossen, die einen Unterdruck bis zu 0,8 bar erzeugen kann. Es entsteht ein gleichmäßiger und intensiver Grenzzonenkontakt zwischen Wunde und Schwamm. Das Wundsekret wird vollständig abgesaugt und bleibt in einem geschlossenen Ableitungssystem. Ein ideal feuchtes Wundmilieu

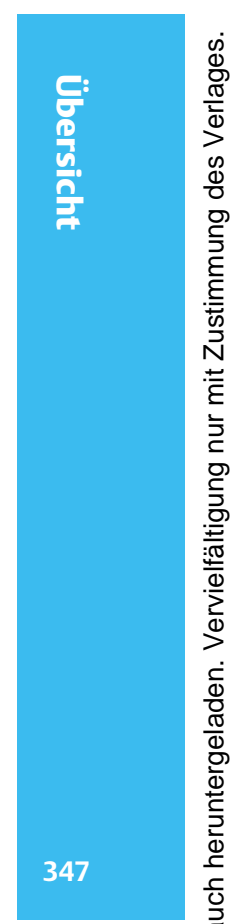


mit konstanter Temperatur beschleunigt die Heilungsvorgänge. Das Ergebnis ist die rasche Bildung eines gesunden, straffen und gefäßreichen Granulationsgewebes; eine ideale Basis für die spontane Epithelisierung oder für plastische Sekundärmaßnahmen. Die Vakuumversiegelung ist absolut undurchlässig für Bakterien, so dass bei traumatischen Wunden eine wirksame Infektprophylaxe erfolgt. Bei septischen Wunden wird eine Keimverteilung in der Patientenumgebung verhindert, das Risiko von Kreuzinfektionen gesenkt und ein positiver Einfluss auf die Krankenhaushygiene ausgeübt.

Die Wirksamkeit der Methode für die lokale Infektbehandlung wird durch die Erweiterung zur Instillations-Vakuum-Versiegelung erhöht. Intermittierend erfolgt durch Installation von antiseptischen oder antibiotischen Lösungen die Umwandlung des Schwamms in einen Medikamententräger. Da Verbandswechsel nur in Abständen von einigen Tagen bis zu einer Woche erforderlich sind, handelt es sich bei der Vakuumversiegelung um eine kostengünstige und patientenfreundliche Behandlungsmöglichkeit von akuten und chronischen Problemwunden $[8,9,12]$.

\section{Biologische Hautäquivalente}

Neben autologer Spalthauttransplantation stehen heutzutage vitale Hautsubstitute mit großer Ähnlichkeit zu menschlicher Haut zum oberflächlichen Wundverschluss zur Verfügung. Es können die drei Formen Epidermisäquivalente, Dermissubstitute
Tab. 3 Biologische Hautäquivalente

$\begin{array}{ll}\text { Epidermisäquivalente } & \text { z. B. EpiDex }{ }^{\mathrm{TM}} \text { (BioCare) } \\ \text { Dermisäquivalente } & \begin{array}{l}\text { z. B. Suprasorb }{ }^{\circledR} \mathrm{C} \\ \text { (Lohmann \& Rauscher) }\end{array} \\ \text { kombinierte Epidermis-Dermis-Äquivalente } & \begin{array}{l}\text { z. B. Graftskin } \\ \text { (Organogenesis Inc.) }\end{array}\end{array}$

und kombinierte Epidermis-Dermis-Äquivalente unterschieden werden (Tab.3). Während der Einsatz nativen Kollagens und von Keratinozytenkulturen bereits weiter verbreitet ist, stellen kombinierte Epidermis-Dermis-Äquivalente eine modernste Variante dar $[5,7,18,22]$.

In Zeiten von boviner spongiformer Enzephalopathie (BSE) muss jedoch der Einsatz dieser Produkte kritisch hinterfragt werden.

\section{Spezielles Wundheilungskonzept beim Dekubitalullkus}

(Abb. 5)

Druckentlastung und Beseitigung einer katabolen Stoffwechsellage sind von entscheidender Bedeutung für den Therapieerfolg. Eine dekompensierte Herzinsuffizienz prädisponiert ebenso zu einem Dekubitus wie eine arterielle Verschlusskrankheit vom Beckentyp. Basis der Dekubitusbehandlung ist die Patientenla-

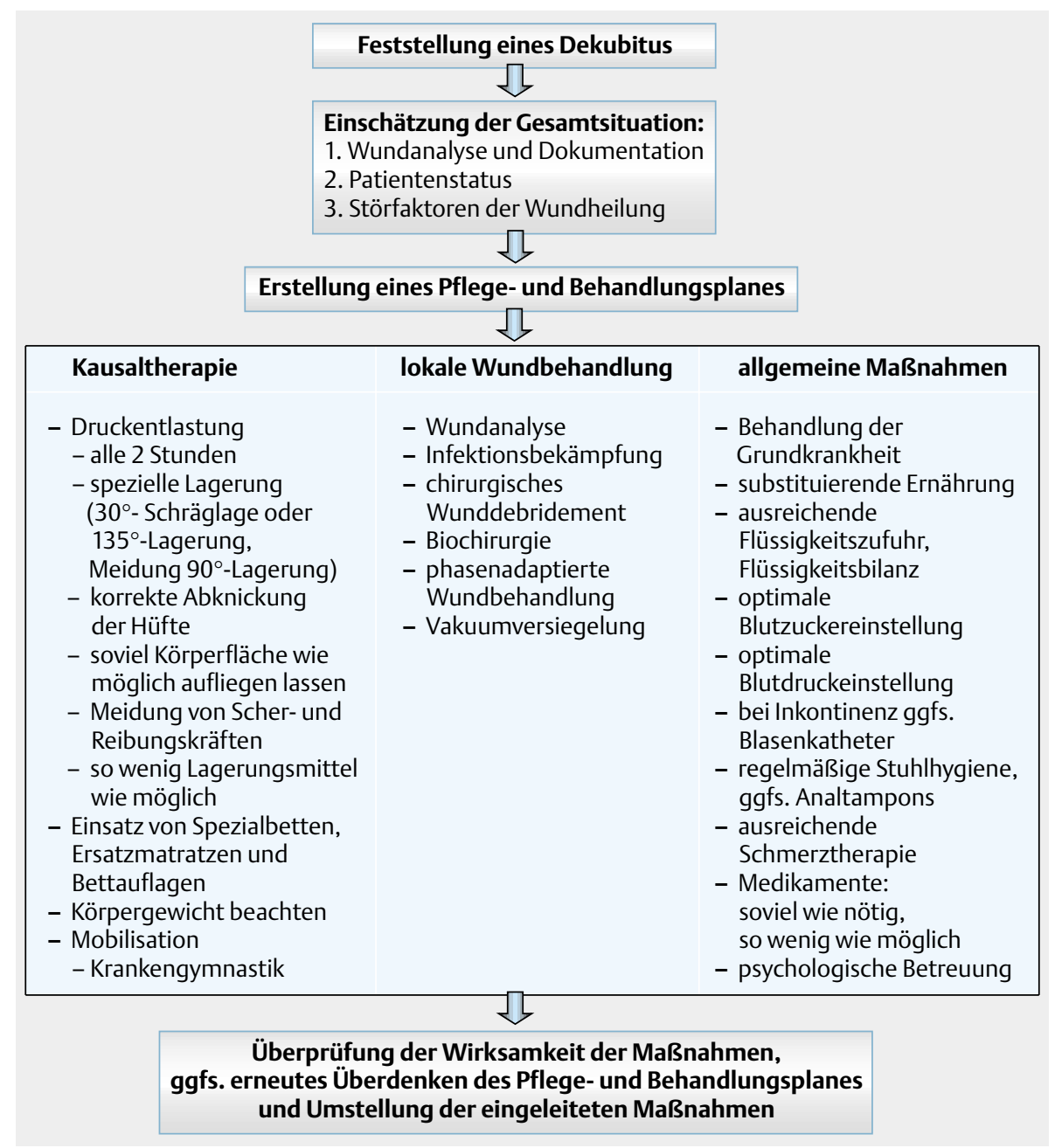

Abb. 5 Management beim Dekubitalulkus. 
Tab. 4 Stadieneinteilung des Dekubitus

\begin{tabular}{ll}
\hline Stadium & Beschreibung \\
\hline I & $\begin{array}{l}\text { persistierende, umschriebene Hautrötung, ggfs. Ödem, } \\
\text { Verhärtung oder Überwärmung (intakte Haut) }\end{array}$ \\
\hline II & $\begin{array}{l}\text { Blase, Hautabschürfung oder flaches Ulkus } \\
\text { (Schädigung der Epidermis bis hin zu Anteilen der Dermis) }\end{array}$ \\
III & $\begin{array}{l}\text { tiefe Ulzeration mit entzündlicher Umbaureaktion } \\
\text { (Schädigung oder Nekrose des subkutanen Gewebes, } \\
\text { die bis zur Faszie reichen kann) } \\
\text { ausgedehnte tiefe Ulzeration unter Einbeziehung von Muskeln, } \\
\text { Knochen, Sehnen oder Gelenkkapsel } \\
\text { (ausgedehnte Gewebsnekrose über die Faszie hinaus) }\end{array}$ \\
\hline
\end{tabular}

Tab. 5 Störfaktoren der Wundheilung beim Dekubitus

\begin{tabular}{|ll|}
\hline systemische Einflüsse & lokale Faktoren \\
\hline schlechter Allgemeinzustand & Gewebshypoxie \\
\hline Mangelernährung & Nekrosen \\
\hline Stoffwechselerkrankung & Infektion \\
\hline Multimorbidität & ungeeigneter Wundverband \\
\hline fortgeschrittenes Alter & \\
\hline herabgesetzter Immunstatus & \\
\hline Medikamente: Zytostatika, Kortikosteroide & \\
\hline Fieber, Exsikkose & \\
\hline Immobilität & \\
\hline Inkontinenz & \\
\hline chronische Schmerzen & \\
\hline mangelnde Mitarbeit des Patienten & \\
\hline
\end{tabular}

gerung auf einer Spezialmatratze, die eine intermittierende Druckentlastung exponierter Körperregionen sicherstellt. Dabei sollte auf faltenfreies Liegen und großflächige Aufliegeflächen geachtet werden.

Beim Dekubitus werden vier Stadien unterschieden (Tab. 4).

Stadium I: reversible Hautrötung.

Stadium II: schmerzhafte oberflächliche Blasenbildung, Erosion oder flache Ulzeration.

Stadium III: tiefe Ulzerationen mit entzündlicher Umbaureaktion.

Stadium IV: ausgedehnte tiefe Ulzeration mit Einbeziehung anderer Gewebsstrukturen.

Die Behandlung richtet sich nach dem Schweregrad des Dekubitus. Bei erhaltener Kontinuität der Haut (Stadium I) sind prophylaktische Maßnahmen indiziert, wobei neben der Lagerung ein Hautschutz durch Aufkleben von z. B. hydrokolloiden Wundauflagen oder transparenten Folienverbänden wirksam ist. Ist bereits eine oberflächliche Wunde entstanden (Stadium II), so ist bei sauberen Ulzerationen mit granulations- und epithelisierungsfördernden Wundauflagen (z.B. Hydrokolloide, Hydrogele,
Polyurethanschaumstoffe) eine problemlose Abheilung zu erwarten. Schwierigkeiten treten bei den tiefen Gewebszerstörungen auf (Stadium III und IV). Eine Wundheilung findet erst dann statt, wenn eine Infektion beseitigt und die Nekrosen abgetragen sind. Erfolgreich ist hier vor allem der Einsatz von „Biosurgeons“. Wundtaschen sollten zur Erreichung sauber und glatt begrenzter Wundränder chirurgisch eröffnet werden. Bei Wunden mit stark zerklüfteten, verschmutzten und infizierten Rändern und gleichartigem Wundgrund eignen sich die Alginate als Wundeinlage gut. Therapieresistente Wunden haben häufig ihre Ursache in einer chronischen Begleitostitis. Ein Therapieerfolg ist hier nur bei konsequenter chirurgischer Behandlung zu erwarten, die aus der Ausräumung septischer Knochen- und Weichteilherde besteht. Die nachfolgende Wundkonditionierung richtet sich nach dem Maß der Infektion, Fibrinbelegung, Exsudation, Granulation und Epithelisierung. Zur Abkürzung der Behandlungsdauer bietet sich die Defektdeckung mittels Spalthauttransplantation oder Lappenplastik an.

Der schlechte Allgemeinzustand vieler Patienten (Tab. 5) verbietet allerdings häufig aufwändige invasive Maßnahmen. Hier bietet die Kombination von Biochirurgie mit Vakuumversiegelung eine gute Perspektive zur vollständigen Weichteilsanierung.

\section{Literatur}

${ }^{1}$ Armstrong S, Ruckley C. Use of fibrous dressings in exsuding leg ulcers. J Wound Care 1997; 6: $322-324$

2 Bonn D. Maggot therapy: an alternative of wound healing. The Lancet 2000; 356: 1174

${ }^{3}$ Bowler P, Jones S, Davies B, Coyle E. Infection control properties of some wound dressings. J Wound Care 1999; 8: 499-502

${ }^{4}$ Brehm G, Goos M, Jung E, Zaun GH. Behandlung von Wunden mit Okklusivverbänden: Klinische und bakteriologische Überlegungen. Akt Dermatol 1991; 4: 83-114

${ }^{5}$ Eaglstein W, Iriondo M, Laszlo K. A composite skin substitute (graftskin) for surgical wounds. Dermatol Surg 1995; 21: 839-843

${ }^{6}$ Eich D, Stadler R. Differenzierte Lokaltherapie chronischer Wunden mit modernen Wundauflagen. VASA 1999; $28: 3-9$

${ }^{7}$ Falanga J. Tissue engineering in wound repair. Adv Skin Wound Care 2000; 13: (Suppl 2) 15-19

8 Fleischmann W, Lang E, Russ M. Infektbehandlung durch Vakuumversiegelung. Unfallchirurg 1997; 100: $301-304$

${ }^{9}$ Fleischmann W, Russ M, Westhauser A, Stampehl M. Die Vakuumversiegelung als Trägersystem für gezielte lokale Medikamentenapplikation bei Wundinfektionen. Unfallchirurg 1998; 101: 649-654

${ }^{10}$ Fritsch P, Schuler G. Hydrokolloide Verbände. Hautarzt 1992; 43 : 597-606

${ }^{11}$ Niedner R. Modernes Wundmanagement. Dialog extra Ulcus cruris. Verlag für medizinische Publikationen, 1995

${ }^{12}$ Nuber V, Tilgen W, Dill-Müller D. Defektdeckung eines chronischen Ulcus cruris mixtum durch Spalthauttransplantation nach Vakuumversiegelung. Z ästh op Derm 2001; 2: 30-33

${ }^{13}$ Petres J, Müller R. Passagere Defektdeckung in der Tumorchirurgie der Haut. Z Hautkr 1985; 60: 185-196

${ }^{14}$ Röthel H, Schenk K. Passive und interaktive Wundauflagen - Aufbau, Wirkung und Einsatzbereiche. Phlebol 1995; 24: 56-60

15 Rufli T. Biochirurgie. Haut 2001; 12: 48-52

${ }^{16}$ Sayag J, Meaume S, Bohbot S. Healing properties of calcium alginate dressings. J Wound Care 1996; 5: 357-362

${ }^{17}$ Schmidt K, Otte H-G, Stadler R. Therapie des Ulcus cruris mit synthetischen Wundauflagen. Zeitschr Hautkr 1996; 71: 254-260

${ }^{18}$ Tanczos E, Horch R, Bannasch H, Andree C, Walgenbach KJ, Voigt M, Stark G. Keratinozytentransplantation und Tissue Engineering. Neue Ansätze in der Behandlung chronischer Wunden. Zentralbl Chir 1999; 124: $81-86$

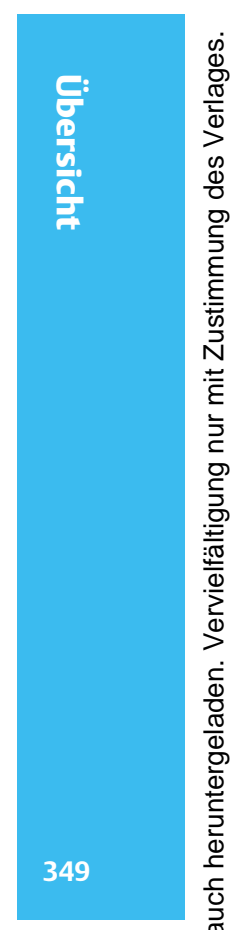


${ }^{19}$ Tebbe B, Orfanos C. Behandlung von Ulcera cruris und Dekubitus mit einem Xerodressing: phasenübergreifende Wundauflage mit antimikrobieller Wirksamkeit. Zeitschr Hautkr 1996; 71: 697-702

${ }^{20}$ Thomas S, Andrews A, Hay N, Bourgoise S. The anti-microbial activity of maggot secretions: results of a preliminary study. J Tissue Viability 1999; 9: $127-132$

${ }^{21}$ Thuro H. Wundbehandlung mit Spezialverbänden. Beilage Hautarzt 1997; 48: $1-4$
22 Veves A, Falanga V, Armstrong D, Sabolinski M. Graftskin, a human skin equivalent, is effective in the management of noninfected neuropathic diabetic foot ulcers. Diabetes Care 2001; 24: 290 - 295

${ }^{23}$ Vloemans A, Kreis R. Fixation of skin grafts with a new silicone rubber dressing (MepitheIR). Scand J Plast Reconstr Hand Surg 1995; 28: $75-76$

${ }^{24}$ Williams C. Tegasorb hydrocolloid dressing: advanced formulation. $\mathrm{Br}$ J Nurs 1996; 5: 1271 - 1272

25 Wollina U, Karte K, Herold C, Looks A. Biosurgery in wound healing the renaissance of maggot therapy. JEADV 2000; 14: 285-289 